\title{
棓RIIMA

\section{A Nash-game approach to joint data completion and location of small inclusions in Stokes flow}

\author{
Marwa OUNI $^{* 1,3}$, Abderrahmane HABBAL ${ }^{1,2}$, Moez KALLEL ${ }^{3}$ \\ ${ }^{1}$ University of Côte d'Azur, LJAD, Parc Valrose 06108 Nice, France \\ ${ }^{2}$ Polytechnic Mohammed VI University, Benguerir, Morocco \\ ${ }^{3}$ University of Tunis El Manar, ENIT-LAMSIN, BP 37, 1002 Tunis-Belvédère, Tunisia \\ *E-mail : marwa.ouni@enit.utm.tn \\ DOI : 10.46298/arima.6761 \\ Submitted on 07 September 2020 - Published on 06 June 2021 \\ Volume : 34 - Year : 2020 \\ Special Issue : Special Issue CARI 2020 \\ Editors : Nabil Gmati, Mathieu Roche, Tri Nguyen-Huu, Laurent Debreu
}

\begin{abstract}
We consider the coupled inverse problem of data completion and the determination of the best locations of an unknown number of small objects immersed in a stationary viscous fluid. We carefully introduce a novel method to solve this problem based on a game theory approach. A new algorithm is provided to recovering the missing data and the number of these objects and their approximate location simultaneously. The detection problem is formulated as a topological one. We present two test-cases that illustrate the efficiency of our original strategy to deal with the ill-posed problem.
\end{abstract}

\section{Keywords}

Geometric inverse problem; Data completion; Calculus of variations; Topological sensitivity; Nash games

\section{INTRODUCTION AND MOTIVATION}

Geometric inverse problems are central in various fields of industrial, biological, and biomedical processes. Challenging theoretical and computational problems arising in fluid mechanics have been intensively investigated, see e.g. [4]. In the present paper, we study a geometric identification problem related to Stokes flows. The problem amounts to determining the unknown number of small objects located in a stationary viscous fluid and their approximate locations, using partial boundary measurements. The latter are of Cauchy type data, available only on the -accessible- part of the outer boundary. Then, the inverse problem under consideration here consists of a coupled inverse problem of object detection and data completion. The identifiability result for the inverse inclusion Cauchy-Stokes problem, with a homogeneous Neumann 
condition imposed on the unknown geometry, of Habbal et al. [8] implies that a single pair of compatible- measurements is enough to recover the unknown objects and the missing boundary data on the remaining -inaccessible- part of the exterior boundary. It is well known that the considered inverse problems are ill-posed, in Hadamard's sense [9]. The problem's ill-posed character is worsened by the coupling, and is critically related to the solution's instability. Illposedness makes classical numerical methods usually inappropriate, and consequently, some regularization techniques have to be used to solve the problem numerically. In order to address the present coupled ill-posedness, we use a game-theoretic framework, following the works $[7,8]$. We then suggest an alternating minimization approach, where the coupled inverse problem is formulated as a three players non-cooperative Nash game. The two first players are associated with the data completion while the third one is in charge of identifying the number of small objects and their location. The small size assumption made on the objects allows deriving an asymptotic expansion of an involved functional. To solve the identification problem, we use the topological gradient notion. Topological sensitivity analysis related to Stokes equations has been investigated in the past by several authors. Various other mathematical approaches in different frameworks are available to solve the obstacle inverse problem, such that the level-set approach, shape gradient method, and homogenization theory.

Let us introduce a preliminary mathematical description of the problem. Consider a bounded open domain $\Omega \subset \mathbb{R}^{d}(\mathrm{~d}=2,3)$, which is filled with a viscous incompressible fluid. We assume that a finite number of the objects included in the domain $\Omega$, and we also suppose that these unknown objects are well separated and have the geometry form: $\mathcal{O}_{z_{k}, \epsilon}=z_{k}+\epsilon \mathcal{B}_{k} ; \forall k \in$ $\{1, \ldots, m\}$, where $\epsilon$ is the shared diameter and $\mathcal{B}_{k}$ is bounded and smooth domain containing the origin. The points $z_{k} \in \Omega$ determine the location of the unknown objects inside $\Omega$. Finally, we suppose that for $k \in\{1, \ldots, m\}$, the object $\mathcal{O}_{z_{k}, \epsilon}$ is far from the exterior boundary $\partial \Omega$, which is composed of two disjoint components $\Gamma_{i}$ and $\Gamma_{c}$, see Figure-1.

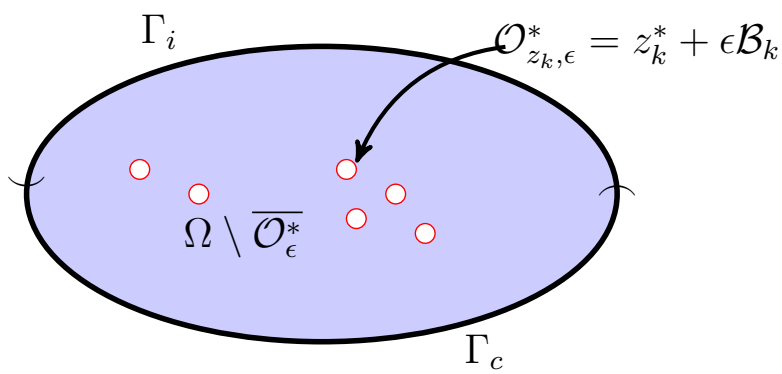

Figure 1: An example of the geometric configuration of the problem: The whole domain $\Omega$, the inclusions $\mathcal{O}_{\epsilon}^{*}$, and two parts of the boundary $\Gamma_{c}$ and $\Gamma_{i}$.

The problem we study then is to detect some small objects (location of the inclusions), from given fluid velocity $f$ and stress forces $\Phi$ prescribed only on the accessible part $\Gamma_{c}$ of the boundary. We denote $\mathcal{O}_{\epsilon}^{*}=\cup_{k=1}^{m} \mathcal{O}_{z_{k}, \epsilon}^{*}$ and we consider the following problem:

$$
\left\{\begin{array}{rllc}
\Delta u-\nabla p & =0 & \text { in } & \Omega \backslash \overline{\mathcal{O}_{\epsilon}^{*}}, \\
\operatorname{div} u & =0 & \text { in } & \Omega \backslash \overline{\mathcal{O}_{\epsilon}^{*}} \\
\sigma(u, p) n & =0 & \text { on } & \partial \mathcal{O}_{\epsilon}^{*}, \\
u & =f & \text { on } & \Gamma_{c} \\
\sigma(u, p) n & =\Phi & \text { on } & \Gamma_{c},
\end{array}\right.
$$


where $u$ denote the velocity filed, $p$ the pressure, and $\sigma(u, p)$ represents the stress tensor defined by:

$$
\sigma(u, p)=2 \nu \mathcal{D}(u)-p I_{d}
$$

with $\nu$ is the kinematic viscosity of the fluid, $\mathcal{D}(u)=1 / 2\left(\nabla u+{ }^{t} \nabla u\right)$ is the deformation tensor, and $I_{d}$ is the identity matrix.

This work aims to recover the missing data of fluid velocity $u$ and stress forces $\sigma(u, p) n$ over $\Gamma_{i}$ the inaccessible part of the boundary from available measurements on the accessible part $\Gamma_{c}$, in addition determine the number of the small objects included in a flow domain and their approximate locations. Section 2 presents an original approach to jointly solving data completion and object detection problems using a Nash game strategy. A topological sensitivity analysis method is used in order to determine the optimal locations of these inclusions, and a new algorithm is provided. In section 3, we illustrate the efficiency of the proposed method by treating two different situations.

\section{DATA COMPLETION AND LOCALIZATION OF SMALL OBJECTS}

Assuming that the Cauchy data $f$ and $\Phi$ belongs to $H^{\frac{1}{2}}\left(\Gamma_{c}\right)^{d} \times\left(H_{00}^{\frac{1}{2}}\left(\Gamma_{c}\right)^{d}\right)^{\prime}$. For given functions $\eta \in\left(H_{00}^{\frac{1}{2}}\left(\Gamma_{i}\right)^{d}\right)^{\prime}, \tau \in H^{\frac{1}{2}}\left(\Gamma_{i}\right)^{d}$, and $\mathcal{O}_{\epsilon}=\cup_{k=1}^{m} z_{k}+\epsilon \mathcal{B}_{k} \subset \Omega$, we define $\left(u_{1}^{\epsilon}, p_{1}^{\epsilon}\right)=$ $\left(u_{1}^{\epsilon}(\eta), p_{1}^{\epsilon}(\eta)\right),\left(u_{2}^{\epsilon}, p_{2}^{\epsilon}\right)=\left(u_{2}^{\epsilon}(\tau), p_{2}^{\epsilon}(\tau)\right)$, and $\left(u_{3}^{\epsilon}, p_{3}^{\epsilon}\right)=\left(u_{3}^{\epsilon}(\tau), p_{3}^{\epsilon}(\tau)\right)$ as the unique weak solutions of the following Stokes mixed boundary value problems:

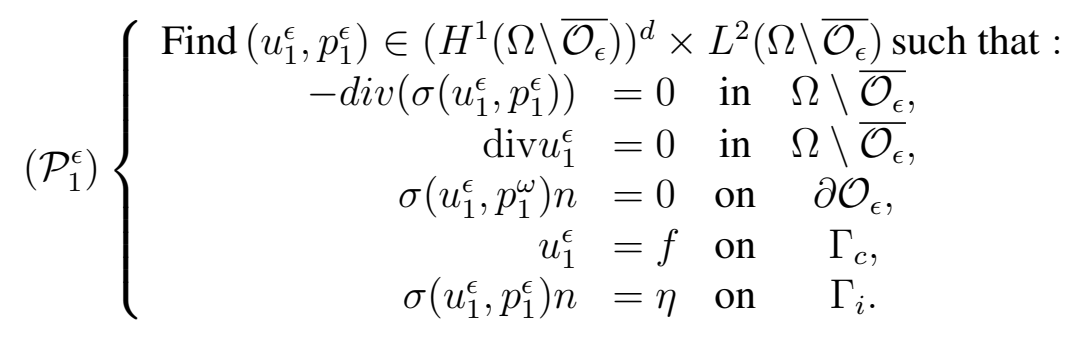

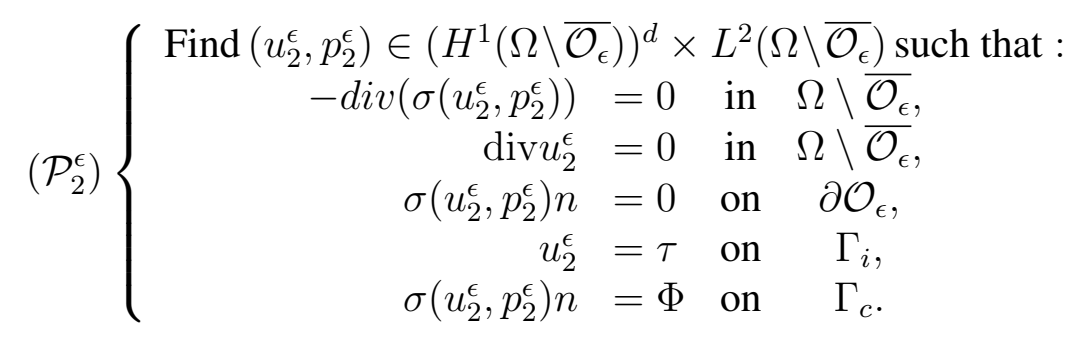

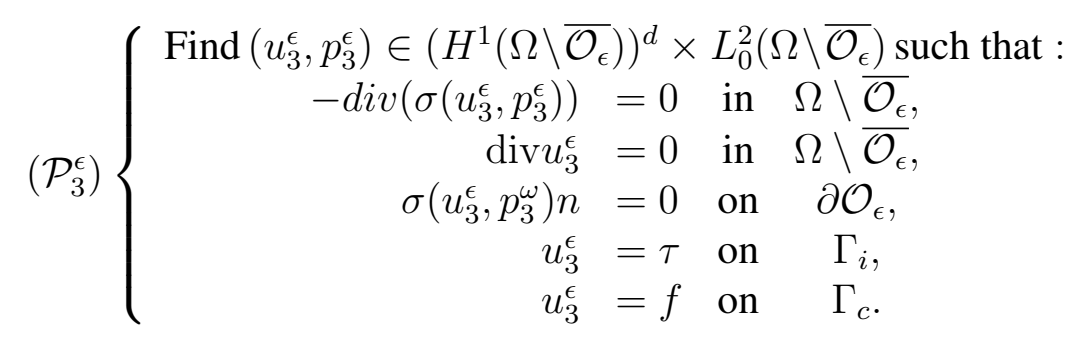

In order to solve the inverse problem (1), we use the simplest class of games. Let us present the 
following three costs: For $(\eta, \tau ; \mathcal{Z}) \in\left(H_{00}^{\frac{1}{2}}\left(\Gamma_{i}\right)^{d}\right)^{\prime} \times H^{\frac{1}{2}}\left(\Gamma_{i}\right)^{d} \times \Omega$,

$$
\begin{aligned}
& \mathcal{J}_{1}(\eta, \tau ; \mathcal{Z})=\frac{1}{2}\left\|\sigma\left(u_{1}^{\epsilon}, p_{1}^{\epsilon}\right) n-\Phi\right\|_{\left(H_{00}^{\left.\frac{1}{2}\left(\Gamma_{c}\right)^{d}\right)^{\prime}}\right.}^{2}+\frac{1}{2}\left\|u_{1}^{\epsilon}-u_{2}^{\epsilon}\right\|_{H^{\frac{1}{2}}\left(\Gamma_{i}\right)^{d}}^{2}, \\
& \mathcal{J}_{2}(\eta, \tau ; \mathcal{Z})=\frac{1}{2}\left\|u_{2}^{\epsilon}-f\right\|_{H^{\frac{1}{2}\left(\Gamma_{c}\right)^{d}}}^{2}+\frac{1}{2} \| u_{1}^{\epsilon}-\left.u_{2}^{\epsilon}\right|_{H^{\frac{1}{2}}\left(\Gamma_{i}\right)^{d}} ^{2}, \\
& \mathcal{J}_{3}(\eta, \tau ; \mathcal{Z})=2 \nu\left\|\mathcal{D}\left(u_{3}^{\epsilon}\right)-\mathcal{D}\left(u_{2}^{\epsilon}\right)\right\|_{L^{2}\left(\Omega\left(\overline{\mathcal{O}_{\epsilon}}\right)\right.}^{2} .
\end{aligned}
$$

We shall say that there are three players: Player 1 and 2 control the respective strategies $\eta \in\left(H_{00}^{\frac{1}{2}}\left(\Gamma_{i}\right)^{d}\right)^{\prime}$ and $\tau \in H^{\frac{1}{2}}\left(\Gamma_{i}\right)^{d}$, and try to minimize their own cost, namely, $\mathcal{J}_{1}$ for Player 1 and $\mathcal{J}_{2}$ for Player 2 , such that each cost functional split into a classical least square term depending only on $\eta$ in $\mathcal{J}_{1}$ and $\tau$ in $\mathcal{J}_{2}$, and a common coupling term $\frac{1}{2}\left\|u_{1}^{\epsilon}-u_{2}^{\epsilon}\right\|_{H^{\frac{1}{2}\left(\Gamma_{i}\right)^{d}}}^{2}$, which depends on both $\eta$ and $\tau$, and has a regularization effect in the partial minimization. Player 3 controls the strategy variable $\mathcal{Z}=\left\{z_{1}, \ldots, z_{m}\right\} \in \Omega$, where no information on the number $m$ is given, and tries to minimize a cost functional $\mathcal{J}_{3}$ of Kohn-Vogelius type, which basically relies on an identifiability result.

We seek the solution of the original coupled problem as a Nash equilibrium, defined as follows:

\section{Definition 1:}

A triplet $\left(\eta_{N}, \tau_{N}, \mathcal{Z}_{N}\right) \in\left(H_{00}^{\frac{1}{2}}\left(\Gamma_{i}\right)^{d}\right)^{\prime} \times H^{\frac{1}{2}}\left(\Gamma_{i}\right)^{d} \times \Omega$ is a Nash equilibrium for the three players game involving the costs $\mathcal{J}_{1}, \mathcal{J}_{2}$ and $\mathcal{J}_{3}$ if:

$$
(N E)\left\{\begin{array}{l}
\mathcal{J}_{1}\left(\eta_{N}, \tau_{N}, \mathcal{Z}_{N}\right) \leqslant \mathcal{J}_{1}\left(\eta, \tau_{N}, \mathcal{Z}_{N}\right), \forall \eta \in\left(H_{00}^{\frac{1}{2}}\left(\Gamma_{i}\right)^{d}\right)^{\prime} \\
\mathcal{J}_{2}\left(\eta_{N}, \tau_{N}, \mathcal{Z}_{N}\right) \leqslant \mathcal{J}_{2}\left(\eta_{N}, \tau, \mathcal{Z}_{N}\right), \forall \tau \in H^{\frac{1}{2}}\left(\Gamma_{i}\right)^{d} \\
\mathcal{J}_{3}\left(\eta_{N}, \tau_{N}, \mathcal{Z}_{N}\right) \leqslant \mathcal{J}_{3}\left(\eta_{N}, \tau_{N}, \mathcal{Z}\right), \forall \mathcal{Z} \in \Omega
\end{array}\right.
$$

Remark 1:

If we consider the case where possible object locations are known, concentrating only on the data completion problem, then the existence of a two-players Nash equilibrium can be proved by using the partial ellipticity of $\mathcal{J}_{1}$ and $\mathcal{J}_{2}$ with respect to $\eta$ and $\tau$, respectively [7, 8]. This property allows us to restrict the search for Nash equilibria to bounded subsets of the strategy spaces, which remains consistent with the classical results of conditional stability of the Cauchy problem [1]. The existence of a three-players Nash equilibrium is much less easy to prove and is out of the scope of the present paper.

\section{Remark 2:}

We sometimes use the improper notation $\mathcal{Z} \in \Omega$ to stipulate that $\mathcal{Z}$ is made of a collection of points in $\Omega$ whose size is unknown.

The minimization problem $\min _{\mathcal{Z} \in \Omega} \mathcal{J}_{3}$ can be formulated as a topological optimization problem as follows: for fixed $\eta \in\left(H_{00}^{\frac{1}{2}}\left(\Gamma_{i}\right)^{d}\right)^{\prime}$ and $\tau \in H^{\frac{1}{2}}\left(\Gamma_{i}\right)^{d}$,

$$
\left(\mathcal{P}_{\epsilon}\right)\left\{\begin{array}{c}
\text { Find } \mathcal{Z}^{*}=\left\{z_{1}^{*}, \ldots, z_{m}^{*}\right\} \in \Omega, \text { such that : } \\
\mathcal{J}\left(\Omega \backslash \overline{\mathcal{O}_{\epsilon}^{*}}\right)=\min _{z_{k} \in \Omega} \mathcal{J}\left(\Omega \backslash \overline{\mathcal{O}_{\epsilon}}\right),
\end{array}\right.
$$


where $\mathcal{O}_{\epsilon}^{*}=\cup_{k=1}^{m} z_{k}^{*}+\epsilon \mathcal{B}_{k} \subset \Omega$, and $\mathcal{J}$ is defined by

$$
\mathcal{J}\left(\Omega \backslash \overline{\mathcal{O}_{\epsilon}}\right)=\mathcal{J}_{K V}\left(u_{2}^{\epsilon}, u_{3}^{\epsilon}\right):=\mathcal{J}_{3}(\eta, \tau ; \mathcal{Z})
$$

In order to solve the optimization problem $\left(\mathcal{P}_{\epsilon}\right)$ above, we recourse to the topological gradient method. The topological gradient method has been known as an efficient approach to solving geometric optimization problems. It consists of studying the variation of a cost function with respect to modifying the topology of the domain $\Omega$. For simplicity in what follows, we will consider the case of a single object $\mathcal{O}_{z, \epsilon}$. Notice that in the case of several inclusions, the results presented below are still valid.

\subsection{The topological gradient method}

The topological sensitivity analysis consists in the study of the variations of a design functional $\mathcal{J}$ with respect to the insertion of a small inclusion $\mathcal{O}_{z, \epsilon}$ at the point $z$. Then, an asymptotic expansion of the function $\mathcal{J}$ can be obtained in the following form: for $\epsilon>0$

$$
\begin{aligned}
\mathcal{J}\left(\Omega_{z, \epsilon}\right)= & \mathcal{J}(\Omega)+\rho(\epsilon) \delta \mathcal{J}(z)+o(\rho(\epsilon)), \forall z \in \Omega \\
& \lim _{\epsilon \rightarrow 0} \rho(\epsilon)=0, \rho(\epsilon)>0
\end{aligned}
$$

where $\Omega_{z, \epsilon}=\Omega \backslash \overline{\mathcal{O}_{z, \epsilon}}$ and the function $\delta \mathcal{J}(z)$ is the so-called topological gradient. This function $\delta \mathcal{J}(z)$ provides an information for creating a small hole located at $z$. Hence, it can be used like a descent direction in an optimization process. Therefore, to minimize the cost function, one has to create small hole at the location $z$ when $\delta \mathcal{J}$ is the most negative.

The concept of the topological derivative was introduced by Schumacher et al [5] in the case of compliance minimization. Next, Sokolowski et al [12] extended it to a more general shape functionals. Various kinds of topology optimization problems have been solved efficiently by using the topological gradient method. It has been widely applied in literature for arbitrarily shaped perturbations and a general class of cost functionals related to PDEs : the elasticity equations [6], Laplace equations [2], Maxwell equations [11], and Stokes system [4], with a homogeneous boundary conditions prescribed on the boundary of the objects.

An adaptation of the adjoint method to the topological context is developed in [6]. We now present the following proposition that describes a generalized adjoint method for the computation of the first variation of a given cost functional.

\section{Proposition 1:}

Let $\mathcal{V}$ be a Hilbert space. For $\epsilon \in[0, \xi), \xi \geqslant 0$, consider a function $u_{\epsilon} \in \mathcal{V}$ that's solution of a variational problem of the form

$$
\mathcal{A}_{\epsilon}\left(u_{\epsilon}, v\right)=l_{\epsilon}(v), \quad \forall v \in \mathcal{V}
$$

where $\mathcal{A}_{\epsilon}$ and $l_{\epsilon}$ are a bilinear form and a linear form on $\mathcal{V}$, respectively. For all $\epsilon \in[0, \xi)$, consider a functional $j(\epsilon)=J_{\epsilon}\left(u_{\epsilon}\right)$, where $J_{\epsilon}$ is Frêchet differentiable at the point $u_{0}$ and its derivative being denoted $D J\left(u_{0}\right)$. Suppose that the following hypotheses hold: 
(i)- There exist two numbers $\delta a, \delta l$ and a function $\rho(\epsilon) \geqslant 0$ such that

$$
\begin{gathered}
\left(\mathcal{A}_{\epsilon}-\mathcal{A}_{0}\right)\left(u_{0}, v_{\epsilon}\right)=\rho(\epsilon) \delta a+o(\rho(\epsilon)), \\
\left(l_{\epsilon}-l_{0}\right)\left(v_{\epsilon}\right)=\rho(\epsilon) \delta l+o(\rho(\epsilon)), \\
\lim _{\epsilon \rightarrow 0} \rho(\epsilon)=0
\end{gathered}
$$

where $v_{\epsilon}$ is an adjoint state satisfying,

$$
\mathcal{A}_{\epsilon}\left(w, v_{\epsilon}\right)=-D J\left(u_{0}\right) w, \forall w \in \mathcal{V} \text {. }
$$

(ii)- There exist a real number $\delta J$ such that

$$
J_{\epsilon}\left(u_{\epsilon}\right)=J_{0}\left(u_{0}\right)+D J\left(u_{0}\right)\left(u_{\epsilon}-u_{0}\right)+\rho(\epsilon) \delta J+o(\rho(\epsilon)) .
$$

Then, the first variation of the cost function with respect to $\epsilon$ is given by

$$
j(\epsilon)=j(0)+\rho(\epsilon)(\delta a+\delta l+\delta J)+o(\rho(\epsilon)) .
$$

\subsection{Application to the model problem}

The aim here is to derive an asymptotic expansion for our functional $\mathcal{J}$ defined in (5) following the same steps described in the proposition 1 above. Then, we shall give explicitly the variations $\delta a, \delta l, \delta \mathcal{J}_{K V}$.

We start by defining the -control free- Sobolev state spaces : Given $g \in H^{\frac{1}{2}}\left(\Gamma_{i}\right)^{d}$ and $\phi \in$ $H^{\frac{1}{2}}(\partial \Omega)$

$$
\mathcal{V}_{1, g}^{\epsilon}=\left\{v \in H^{1}\left(\Omega_{z, \epsilon}\right)^{d} \text {, such that } \operatorname{div} v=0 \text { in } \Omega_{z, \epsilon} \text { and } v_{\left.\right|_{\Gamma_{i}}}=g\right\}
$$

and

$$
\mathcal{V}_{2, \phi}^{\epsilon}=\left\{v \in H^{1}\left(\Omega_{z, \epsilon}\right)^{d}, \text { such that } \operatorname{div} v=0 \text { in } \Omega_{z, \epsilon} \text { and } v_{\left.\right|_{\partial \Omega}}=\phi\right\}
$$

The variational formulations associated to problems $\left(\mathcal{P}_{2}^{\epsilon}\right)$ and $\left(\mathcal{P}_{3}^{\epsilon}\right)$ can be stated respectively as follows:

$$
\begin{aligned}
& \| \text { Find } u_{2}^{\epsilon} \in \mathcal{V}_{1, \tau}^{\epsilon} \text { such that : } \\
& \mathcal{A}_{1, \epsilon}\left(u_{2}^{\epsilon}, v\right)=l_{1, \epsilon}(v), \forall v \in \mathcal{V}_{1,0}^{\epsilon},
\end{aligned}
$$

$$
\begin{aligned}
& \text { Find } u_{3}^{\epsilon} \in W=\left\{\phi \in \mathcal{V}_{1, \tau}^{\epsilon} / \phi_{\left.\right|_{\Gamma_{c}}}=f\right\} \text { such that : } \\
& \mathcal{A}_{2, \epsilon}\left(u_{3}^{\epsilon}, v\right)=l_{2, \epsilon}(v), \forall v \in \mathcal{V}_{2,0}^{\epsilon},
\end{aligned}
$$

where

$$
\begin{aligned}
& \mathcal{A}_{1, \epsilon}\left(u_{2}^{\epsilon}, v\right)=2 \nu \int_{\Omega_{z, \epsilon}} \mathcal{D}\left(u_{2}^{\epsilon}\right): \mathcal{D}(v) d x \\
& \mathcal{A}_{2, \epsilon}\left(u_{3}^{\epsilon}, v\right)=2 \nu \int_{\Omega_{z, \epsilon}} \mathcal{D}\left(u_{3}^{\epsilon}\right): \mathcal{D}(v) d x
\end{aligned}
$$




$$
l_{1, \epsilon}(v)=\int_{\Gamma_{c}} \Phi v d s
$$

and

$$
l_{2, \epsilon}(v)=0 .
$$

Note that for $\epsilon=0$, we have $\Omega_{0}=\Omega$, and $\left(u_{2}^{0}, p_{2}^{0}\right) \in H^{1}(\Omega)^{d} \times L^{2}(\Omega)$ and $\left(u_{3}^{0}, p_{3}^{0}\right) \in H^{1}(\Omega)^{d} \times$ $L_{0}^{2}(\Omega)$ solve the respective boundary value problems:

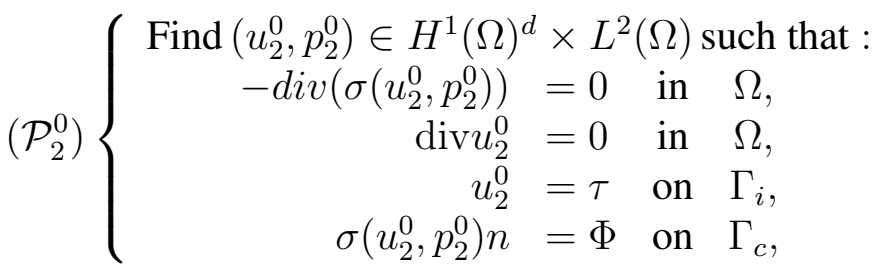

$$
\begin{aligned}
& \left(\mathcal{P}_{3}^{0}\right)\left\{\begin{aligned}
\text { Find }\left(u_{3}^{0}, p_{3}^{0}\right) \in H^{1}(\Omega)^{d} & \times L_{0}^{2}(\Omega) \text { such that : } \\
-\operatorname{div}\left(\sigma\left(u_{3}^{0}, p_{3}^{0}\right)\right) & =0 \quad \text { in } \Omega, \\
\operatorname{div} u_{3}^{0} & =0 \text { in } \Omega, \\
u_{3}^{0} & =\tau \text { on } \Gamma_{i}, \\
u_{3}^{0} & =f \text { on } \Gamma_{c} .
\end{aligned}\right.
\end{aligned}
$$

VARIATION OF THE BILINEAR FORM $\mathcal{A}_{1, \epsilon}$ AND $\mathcal{A}_{2, \epsilon}$ :

In order to obtain an asymptotic expansion of the variation of the bilinear form, we will use a simplified technique proposed in [4] for the Stokes system. We can also use a truncation technique, which is developed in [6] for elasticity equations, with a Neumann boundary condition on $\partial \mathcal{O}_{z, \epsilon}$.

Variation of $\mathcal{A}_{1, \epsilon}:$ We are interested in the asymptotic analysis of the variation

$$
\begin{aligned}
\left(\mathcal{A}_{1, \epsilon}-\mathcal{A}_{1,0}\right)\left(u_{2}^{0}, v_{1}^{\epsilon}\right) & =-\mathcal{A}_{1, \epsilon}\left(u_{2}^{\epsilon}-u_{2}^{0}, v_{1}^{\epsilon}\right)+\left(l_{1, \epsilon}\left(v_{1}^{\epsilon}\right)-l_{1,0}\left(v_{1}^{\epsilon}\right)\right) \\
& =-\mathcal{A}_{1, \epsilon}\left(u_{2}^{\epsilon}-u_{2}^{0}, v_{1}^{\epsilon}\right) \\
& =-2 \nu \int_{\Omega_{z, \epsilon}} \mathcal{D}\left(u_{2}^{\epsilon}-u_{2}^{0}\right): \mathcal{D}\left(v_{1}^{\epsilon}\right) d x \\
& =\int_{\partial \mathcal{O}_{\mathrm{z}, \epsilon}} \sigma\left(u_{2}^{0}, p_{2}^{0}\right) n v_{1}^{\epsilon} d s .
\end{aligned}
$$

According to fundamental assumption (i) carefully formulated in proposition 1, we search to find a real number $\delta a_{1} \in \mathbb{R}$ and a scalar function positive $\rho$ such that

$$
\begin{gathered}
\int_{\partial \mathcal{O}_{\mathrm{z}, \epsilon}} \sigma\left(u_{2}^{0}, p_{2}^{0}\right) n v_{1}^{\epsilon} d s=\rho(\epsilon) \delta a_{1}+o(\rho(\epsilon)), \\
\lim _{\epsilon \rightarrow 0} \rho(\epsilon)=0 .
\end{gathered}
$$


To this end, we start by splitting the integral above,

$$
\begin{aligned}
\int_{\partial \mathcal{O}_{\mathrm{z}, \epsilon}} \sigma\left(u_{2}^{0}, p_{2}^{0}\right) n v_{1}^{\epsilon} d s & =\int_{\partial \mathcal{O}_{\mathrm{z}, \epsilon}} \sigma\left(u_{2}^{0}, p_{2}^{0}\right) n v_{1}^{0} d s+\int_{\partial \mathcal{O}_{\mathrm{z}, \epsilon}} \sigma\left(u_{2}^{0}, p_{2}^{0}\right) n\left(v_{1}^{\epsilon}-v_{1}^{0}\right) d s \\
& =\mathcal{I}_{1}+\mathcal{I}_{2} .
\end{aligned}
$$

Next, properly using the obtained estimates by Ben Abda et al [4] for each term $\mathcal{I}_{1}$ and $\mathcal{I}_{2}$, which are written as follows

$$
\mathcal{I}_{1}=-2 \nu \epsilon^{2}|\mathcal{B}| \mathcal{D}\left(u_{2}^{0}\right)(z): \mathcal{D}\left(v_{1}^{0}\right)(z)+o\left(\epsilon^{2}\right)
$$

and

$$
\mathcal{I}_{2}=2 \nu \epsilon^{2}|\mathcal{B}| \mathcal{D}\left(u_{2}^{0}\right)(z): \mathcal{D}\left(v_{1}^{0}\right)(z)-\epsilon^{2} \mathcal{D}\left(u_{2}^{0}\right)(z): \int_{\partial \mathcal{B}} \mu(y) y^{T} d s+o\left(\epsilon^{2}\right),
$$

where $\mu \in H^{-\frac{1}{2}}(\partial \mathcal{B})^{d}$ is the solution to the boundary integral equation: $\forall y \in \partial \mathcal{B}$,

$$
\left.-\frac{\mu(y)}{2}+\int_{\partial \mathcal{B}}\left[2 \nu \mathcal{D}_{y}(E)(x-y) \mu(x)\right) n(y)-P(x-y) \mu(x) n(y)\right] d s(x)=-2 \nu \mathcal{D}\left(v_{1}^{0}\right)(z) n(y),
$$

with $(E, P)$ is the fundamental solution to the stokes system in $\mathbb{R}^{2}$. Therefore, we deduce

$$
\left(\mathcal{A}_{1, \epsilon}-\mathcal{A}_{1,0}\right)\left(u_{2}^{0}, v_{1}^{\epsilon}\right)=-\epsilon^{2} \mathcal{D}\left(u_{2}^{0}\right)(z): \int_{\partial \mathcal{B}} \mu(y) y^{T} d s+o\left(\epsilon^{2}\right)
$$

If $\mathcal{B}=\mathcal{B}(0,1)$, using the same technique as that used in [4], we obtain

$$
\delta a_{1}=-4 \nu \mathcal{D}\left(u_{2}^{0}\right)(z): \mathcal{D}\left(v_{1}^{0}\right)(z),
$$

where $v_{1}^{0} \in \mathcal{V}_{1,0}^{0}$ is the solution to the associated adjoint problem:

$$
\mathcal{A}_{1,0}\left(w, v_{1}^{0}\right)=-\partial_{u_{2}^{0}} \mathcal{J}_{K V}\left(u_{2}^{0}, u_{3}^{0}\right) w, \forall w \in \mathcal{V}_{1,0}^{0} .
$$

Variation of $\mathcal{A}_{2, \epsilon}$ : Let us mention that the same bilinear form is available also for the $\left(\mathcal{P}_{3}^{\epsilon}\right)$, namely, $\mathcal{A}_{1, \epsilon} \equiv \mathcal{A}_{2, \epsilon}$. Thus, the variation of $\mathcal{A}_{1, \epsilon}$ associated to $\left(\mathcal{P}_{3}^{\epsilon}\right)$ is written as follows:

$$
\left(\mathcal{A}_{2, \epsilon}-\mathcal{A}_{2,0}\right)\left(u_{3}^{0}, v_{2}^{\epsilon}\right)=-4 \nu \pi \epsilon^{2} \mathcal{D}\left(u_{3}^{0}\right)(z): \mathcal{D}\left(v_{2}^{0}\right)(z)
$$

where $v_{2}^{0} \in \mathcal{V}_{2,0}^{0}$ is the solution to the associated adjoint problem:

$$
\mathcal{A}_{2,0}\left(w, v_{2}^{0}\right)=-\partial_{u_{3}^{0}} \mathcal{J}_{K V}\left(u_{2}^{0}, u_{3}^{0}\right) w, \forall w \in \mathcal{V}_{2,0}^{0}
$$

VARIATION OF THE LINEAR FORM $l_{1, \epsilon}$ AND $l_{2, \epsilon}$ :

Since $l_{1, \epsilon}$ and $l_{2, \epsilon}$ are independent of $\epsilon$, it follows trivially that $\delta l_{1}=\delta l_{2}=0$. 
VARIATION OF THE COST FUNCTIONAL $\mathcal{J}_{K V}$ :

Let us now turn to the asymptotic analysis of the variation of the Kohn-Vogeluis functional given by

$$
\mathcal{J}_{K V}\left(u_{2}^{\epsilon}, u_{3}^{\epsilon}\right)=2 \nu \int_{\Omega_{z, \epsilon}} \mathcal{D}\left(u_{3}^{\epsilon}-u_{2}^{\epsilon}\right): \mathcal{D}\left(u_{3}^{\epsilon}-u_{2}^{\epsilon}\right) d x
$$

One can decompose this above functional as follows:

$$
\mathcal{J}_{K V}\left(u_{2}^{\epsilon}, u_{3}^{\epsilon}\right)=\mathfrak{J}_{1}\left(u_{2}^{\epsilon}\right)+\mathfrak{J}_{2}\left(u_{3}^{\epsilon}\right)-2 \mathfrak{J}_{12}\left(u_{2}^{\epsilon}, u_{3}^{\epsilon}\right),
$$

where

$$
\begin{gathered}
\mathfrak{J}_{1}\left(u_{2}^{\epsilon}\right)=2 \nu \int_{\Omega_{z, \epsilon}} \mathcal{D}\left(u_{2}^{\epsilon}\right): \mathcal{D}\left(u_{2}^{\epsilon}\right) d x, \\
\mathfrak{J}_{2}\left(u_{3}^{\epsilon}\right)=2 \nu \int_{\Omega_{z, \epsilon}} \mathcal{D}\left(u_{3}^{\epsilon}\right): \mathcal{D}\left(u_{3}^{\epsilon}\right) d x \\
\mathfrak{J}_{12}\left(u_{2}^{\epsilon}, u_{2}^{\epsilon}\right)=2 \nu \int_{\Omega_{z, \epsilon}} \mathcal{D}\left(u_{2}^{\epsilon}\right): \mathcal{D}\left(u_{3}^{\epsilon}\right) d x .
\end{gathered}
$$

Variation of $\mathfrak{J}_{1}$ : The variation of $\mathfrak{J}_{1}$ reads

$$
\begin{aligned}
\mathfrak{J}_{1}\left(u_{2}^{\epsilon}\right)- & \mathfrak{J}_{1}\left(u_{2}^{0}\right)=2 \nu \int_{\Omega_{z, \epsilon}} \mathcal{D}\left(u_{2}^{\epsilon}\right): \mathcal{D}\left(u_{2}^{\epsilon}\right) d x-2 \nu \int_{\Omega} \mathcal{D}\left(u_{2}^{0}\right): \mathcal{D}\left(u_{2}^{0}\right) d x \\
& =2 \nu \int_{\Omega_{z, \epsilon}} \mathcal{D}\left(u_{2}^{\epsilon}-u_{2}^{0}\right): \mathcal{D}\left(u_{2}^{\epsilon}\right) d x+2 \nu \int_{\Omega_{z, \epsilon}} \mathcal{D}\left(u_{2}^{\epsilon}-u_{2}^{0}\right): \mathcal{D}\left(u_{2}^{0}\right) d x \\
& -2 \nu \int_{\mathcal{O}_{\mathrm{z}, \epsilon}} \mathcal{D}\left(u_{2}^{0}\right): \mathcal{D}\left(u_{2}^{0}\right) d x
\end{aligned}
$$

Using the Green formula applied to the problem $\left(\mathcal{P}_{2}^{\epsilon}\right)$, we get

$$
2 \nu \int_{\Omega_{z, \epsilon}} \mathcal{D}\left(u_{2}^{\epsilon}-u_{2}^{0}\right): \mathcal{D}\left(u_{2}^{\epsilon}\right) d x=\int_{\Gamma_{c}} \Phi\left(u_{2}^{\epsilon}-u_{2}^{0}\right) d s .
$$

Then, it follows that

$$
\begin{aligned}
\mathfrak{J}_{1}\left(u_{2}^{\epsilon}\right)-\mathfrak{J}_{1}\left(u_{2}^{0}\right) & =2 \nu \int_{\Omega_{z, \epsilon}} \mathcal{D}\left(u_{2}^{\epsilon}-u_{2}^{0}\right): \mathcal{D}\left(u_{2}^{0}\right) d x-2 \nu \int_{\mathcal{O}_{\mathrm{z}, \epsilon}} \mathcal{D}\left(u_{2}^{0}\right): \mathcal{D}\left(u_{2}^{0}\right) d x \\
& +\int_{\Gamma_{c}} \Phi\left(u_{2}^{\epsilon}-u_{2}^{0}\right) d s
\end{aligned}
$$

Variation of $\mathfrak{J}_{2}$ : The variation of $\mathfrak{J}_{2}$ reads

$$
\begin{aligned}
\mathfrak{J}_{2}\left(u_{3}^{\epsilon}\right)- & \mathfrak{J}_{2}\left(u_{3}^{0}\right)=2 \nu \int_{\Omega_{z, \epsilon}} \mathcal{D}\left(u_{3}^{\epsilon}\right): \mathcal{D}\left(u_{3}^{\epsilon}\right) d x-2 \nu \int_{\Omega} \mathcal{D}\left(u_{3}^{0}\right): \mathcal{D}\left(u_{3}^{0}\right) d x \\
& =2 \nu \int_{\Omega_{z, \epsilon}} \mathcal{D}\left(u_{3}^{\epsilon}-u_{3}^{0}\right): \mathcal{D}\left(u_{3}^{\epsilon}\right) d x+2 \nu \int_{\Omega_{z, \epsilon}} \mathcal{D}\left(u_{3}^{\epsilon}-u_{3}^{0}\right): \mathcal{D}\left(u_{3}^{0}\right) d x \\
& -2 \nu \int_{\mathcal{O}_{z, \epsilon}} \mathcal{D}\left(u_{3}^{0}\right): \mathcal{D}\left(u_{3}^{0}\right) d x
\end{aligned}
$$


Using the Green formula, one can get from $\left(\mathcal{P}_{3}^{\epsilon}\right)$ that

$$
2 \nu \int_{\Omega_{z, \epsilon}} \mathcal{D}\left(u_{3}^{\epsilon}-u_{3}^{0}\right): \mathcal{D}\left(u_{3}^{\epsilon}\right) d x=0 .
$$

Then, we obtain

$$
\mathfrak{J}_{2}\left(u_{2}^{\epsilon}\right)-\mathfrak{J}_{2}\left(u_{2}^{0}\right)=2 \nu \int_{\Omega_{z, \epsilon}} \mathcal{D}\left(u_{3}^{\epsilon}-u_{3}^{0}\right): \mathcal{D}\left(u_{3}^{0}\right) d x-2 \nu \int_{\mathcal{O}_{\mathrm{z}, \epsilon}} \mathcal{D}\left(u_{3}^{0}\right): \mathcal{D}\left(u_{3}^{0}\right) d x .
$$

Variation of $\mathfrak{J}_{12}$ : The variation of $\mathfrak{J}_{12}$ reads

$$
\mathfrak{J}_{12}\left(u_{2}^{\epsilon}, u_{3}^{\epsilon}\right)-\mathfrak{J}_{12}\left(u_{2}^{0}, u_{3}^{0}\right)=2 \nu \int_{\Omega_{z, \epsilon}} \mathcal{D}\left(u_{2}^{\epsilon}\right): \mathcal{D}\left(u_{3}^{\epsilon}\right) d x-2 \nu \int_{\Omega} \mathcal{D}\left(u_{2}^{0}\right): \mathcal{D}\left(u_{3}^{0}\right) d x
$$

Using the Green formula applied to $\left(\mathcal{P}_{2}^{\epsilon}\right)$ and $\left(\mathcal{P}_{2}^{0}\right)$, we obtain

$$
\begin{gathered}
2 \nu \int_{\Omega_{z, \epsilon}} \mathcal{D}\left(u_{2}^{\epsilon}\right): \mathcal{D}\left(u_{3}^{\epsilon}\right) d x=\int_{\Gamma_{c}} \Phi f d s+\int_{\Gamma_{i}} \sigma\left(u_{2}^{\epsilon}, p_{2}^{\epsilon}\right) n \tau d s . \\
2 \nu \int_{\Omega} \mathcal{D}\left(u_{2}^{0}\right): \mathcal{D}\left(u_{3}^{0}\right) d x=\int_{\Gamma_{c}} \Phi f d s+\int_{\Gamma_{i}} \sigma\left(u_{2}^{0}, p_{2}^{0}\right) n \tau d s .
\end{gathered}
$$

Then, we deduce

$$
\mathfrak{J}_{12}\left(u_{2}^{\epsilon}, u_{3}^{\epsilon}\right)-\mathfrak{J}_{12}\left(u_{2}^{0}, u_{3}^{0}\right)=\int_{\Gamma_{i}} \sigma\left(u_{2}^{\epsilon}-u_{2}^{0}, p_{2}^{\epsilon}-p_{2}^{0}\right) n \tau d s .
$$

Combining the variation (8), (9) and (10), the variation of the functional $\mathcal{J}_{K V}$ becomes

$$
\begin{aligned}
\mathcal{J}_{K V}\left(u_{2}^{\epsilon}, u_{3}^{\epsilon}\right) & -\mathcal{J}_{K V}\left(u_{2}^{0}, u_{3}^{0}\right)=-2 \nu \int_{\mathcal{O}_{\mathrm{z}, \epsilon}} \mathcal{D}\left(u_{2}^{0}\right): \mathcal{D}\left(u_{2}^{0}\right) d x-2 \nu \int_{\mathcal{O}_{\mathrm{z}, \epsilon}} \mathcal{D}\left(u_{3}^{0}\right): \mathcal{D}\left(u_{3}^{0}\right) d x \\
& +2 \nu \int_{\Omega_{z, \epsilon}} \mathcal{D}\left(u_{2}^{\epsilon}-u_{2}^{0}\right): \mathcal{D}\left(u_{2}^{0}\right) d x+2 \nu \int_{\Omega_{z, \epsilon}} \mathcal{D}\left(u_{3}^{\epsilon}-u_{3}^{0}\right): \mathcal{D}\left(u_{3}^{0}\right) d x \\
& +\int_{\Gamma_{c}} \Phi\left(u_{2}^{\epsilon}-u_{2}^{0}\right) d s-2 \int_{\Gamma_{i}} \sigma\left(u_{2}^{\epsilon}-u_{2}^{0}, p_{2}^{\epsilon}-p_{2}^{0}\right) n \tau d s .
\end{aligned}
$$

Then for $\epsilon \in[0, \xi)$, we get

$$
\begin{gathered}
D \mathcal{J}_{K V}\left(u_{2}^{0}, u_{3}^{0}\right)\left(u_{2}^{\epsilon}-u_{2}^{0}, u_{3}^{\epsilon}-u_{3}^{0}\right)=2 \nu \int_{\Omega_{z, \epsilon}} \mathcal{D}\left(u_{2}^{\epsilon}-u_{2}^{0}\right): \mathcal{D}\left(u_{2}^{0}\right) d x \\
+2 \nu \int_{\Omega_{z, \epsilon}} \mathcal{D}\left(u_{3}^{\epsilon}-u_{3}^{0}\right): \mathcal{D}\left(u_{3}^{0}\right) d x+\int_{\Gamma_{c}} \Phi\left(u_{2}^{\epsilon}-u_{2}^{0}\right) d s \\
\quad-2 \int_{\Gamma_{i}} \sigma\left(u_{2}^{\epsilon}-u_{2}^{0}, p_{2}^{\epsilon}-p_{2}^{0}\right) n \tau d s
\end{gathered}
$$

Thus, we have

$$
\begin{array}{r}
\mathcal{J}_{K V}\left(u_{2}^{\epsilon}, u_{3}^{\epsilon}\right)-\mathcal{J}_{K V}\left(u_{2}^{0}, u_{3}^{0}\right)=D \mathcal{J}_{K V}\left(u_{2}^{0}, u_{3}^{0}\right)\left(u_{2}^{\epsilon}-u_{2}^{0}, u_{3}^{\epsilon}-u_{3}^{0}\right) \\
-2 \nu \int_{\mathcal{O}_{\mathrm{z}, \epsilon}} \mathcal{D}\left(u_{2}^{0}\right): \mathcal{D}\left(u_{2}^{0}\right) d x-2 \nu \int_{\mathcal{O}_{\mathrm{z}, \epsilon}} \mathcal{D}\left(u_{3}^{0}\right): \mathcal{D}\left(u_{3}^{0}\right) d x
\end{array}
$$


Next, the second term on the right hand side of (11) may be written as

$$
\begin{aligned}
2 \nu \int_{\mathcal{O}_{z, \epsilon}} \mathcal{D}\left(u_{2}^{0}\right): \mathcal{D}\left(u_{2}^{0}\right) d x & =2 \nu \int_{\mathcal{O}_{z, \epsilon}} \mathcal{D}\left(u_{2}^{0}\right)(z): \mathcal{D}\left(u_{2}^{0}\right)(z) d x \\
& +2 \nu \int_{\mathcal{O}_{z, \epsilon}}\left[\mathcal{D}\left(u_{2}^{0}\right)-\mathcal{D}\left(u_{2}^{0}\right)(z)\right]: \mathcal{D}\left(u_{2}^{0}\right) d x \\
& +2 \nu \int_{\mathcal{O}_{z, \epsilon}} \mathcal{D}\left(u_{2}^{0}\right)(z):\left[\mathcal{D}\left(u_{2}^{0}\right)-\mathcal{D}\left(u_{2}^{0}\right)(z)\right] d x
\end{aligned}
$$

Using the Taylor theorem and the change of variables $x=z+\epsilon y$, we obtain

$$
2 \nu \int_{\mathcal{O}_{z, \epsilon}} \mathcal{D}\left(u_{2}^{0}\right): \mathcal{D}\left(u_{2}^{0}\right) d x=2 \nu \epsilon^{2}|\mathcal{B}| \mathcal{D}\left(u_{2}^{0}\right)(z): \mathcal{D}\left(u_{2}^{0}\right)(z)+o\left(\epsilon^{2}\right) .
$$

The same way for the third term, we have

$$
2 \nu \int_{\mathcal{O}_{\mathrm{z}, \epsilon}} \mathcal{D}\left(u_{3}^{0}\right): \mathcal{D}\left(u_{3}^{0}\right) d x=2 \nu \epsilon^{2}|\mathcal{B}| \mathcal{D}\left(u_{3}^{0}\right)(z): \mathcal{D}\left(u_{3}^{0}\right)(z)+o\left(\epsilon^{2}\right) .
$$

Thus,

$$
\delta \mathcal{J}_{K V}=-2 \nu \pi\left(\left|\mathcal{D}\left(u_{2}^{0}\right)(z)\right|^{2}+\left|\mathcal{D}\left(u_{3}^{0}\right)(z)\right|^{2}\right)
$$

Now, we are ready to give the main result of this paper.

\section{Theorem 1:}

If $d=2$, the function $\mathcal{J}$ has the following asymptotic expansion

$$
\mathcal{J}\left(\Omega_{z, \epsilon}\right)-\mathcal{J}(\Omega)=\pi \epsilon^{d}\left(\delta a_{1}\left(u_{2}^{0}, v_{1}^{0}\right)+\delta a_{2}\left(u_{3}^{0}, v_{2}^{0}\right)+\delta \mathcal{J}_{K V}\left(u_{2}^{0}, u_{3}^{0}\right)\right)+o\left(\epsilon^{d}\right),
$$

where $\forall z \in \Omega$, we have

$$
\left\{\begin{aligned}
\delta a_{1}\left(u_{2}^{0}, v_{1}^{0}\right) & =-4 \nu \mathcal{D}\left(u_{2}^{0}\right)(z): \mathcal{D}\left(v_{1}^{0}\right)(z) \\
\delta a_{2}\left(u_{3}^{0}, v_{2}^{0}\right) & =-4 \nu \mathcal{D}\left(u_{3}^{0}\right)(z): \mathcal{D}\left(v_{2}^{0}\right)(z) \\
\delta \mathcal{J}_{K V}\left(u_{2}^{0}, u_{3}^{0}\right) & =-2\left(\left|\mathcal{D}\left(u_{2}^{0}\right)(z)\right|^{2}+\left|\mathcal{D}\left(u_{3}^{0}\right)(z)\right|^{2}\right)
\end{aligned}\right.
$$

with $v_{1}^{0} \in \mathcal{V}_{1,0}^{0}$ and $v_{2}^{0} \in \mathcal{V}_{2,0}^{0}$ are solutions to the adjoint equations associated respectively to the $\left(\mathcal{P}_{2}^{0}\right)$ and $\left(\mathcal{P}_{3}^{0}\right)$ :

$$
\begin{aligned}
& \mathcal{A}_{1,0}\left(w, v_{1}^{0}\right)=-\partial_{u_{2}^{0}} \mathcal{J}\left(u_{2}^{0}, u_{3}^{0}\right) w, \forall w \in \mathcal{V}_{1,0}^{0}, \\
& \mathcal{A}_{2,0}\left(w, v_{2}^{0}\right)=-\partial_{u_{3}^{0}} \mathcal{J}\left(u_{2}^{0}, u_{3}^{0}\right) w, \forall w \in \mathcal{V}_{2,0}^{0} .
\end{aligned}
$$

We now present a novel algorithm dedicated to the Nash equilibrium computation, which is described below. The algorithm is divided into two main steps: In the first, Player 3 seeks to identify the number and the best locations of the objects by minimizing $\mathcal{J}_{3}$ and using a oneshot algorithm based on a topological derivative. In the second one, players 1 and 2 play a Nash subgame in order to precondition the Cauchy problem and tackle its ill-posedness. To save overall computation time, we consider parallel implementations for the computation of the two-players Nash equilibrium based on the alternating minimization algorithm introduced in [3]; we can also assume that the players solve their partial optimization problems sequentially, 
not simultaneously. Those algorithms are similar to alternating and parallel Schwarz methods. A fixed-step gradient method is used to solve the partial optimization problems of $\mathcal{J}_{1}$ and $\mathcal{J}_{2}$.

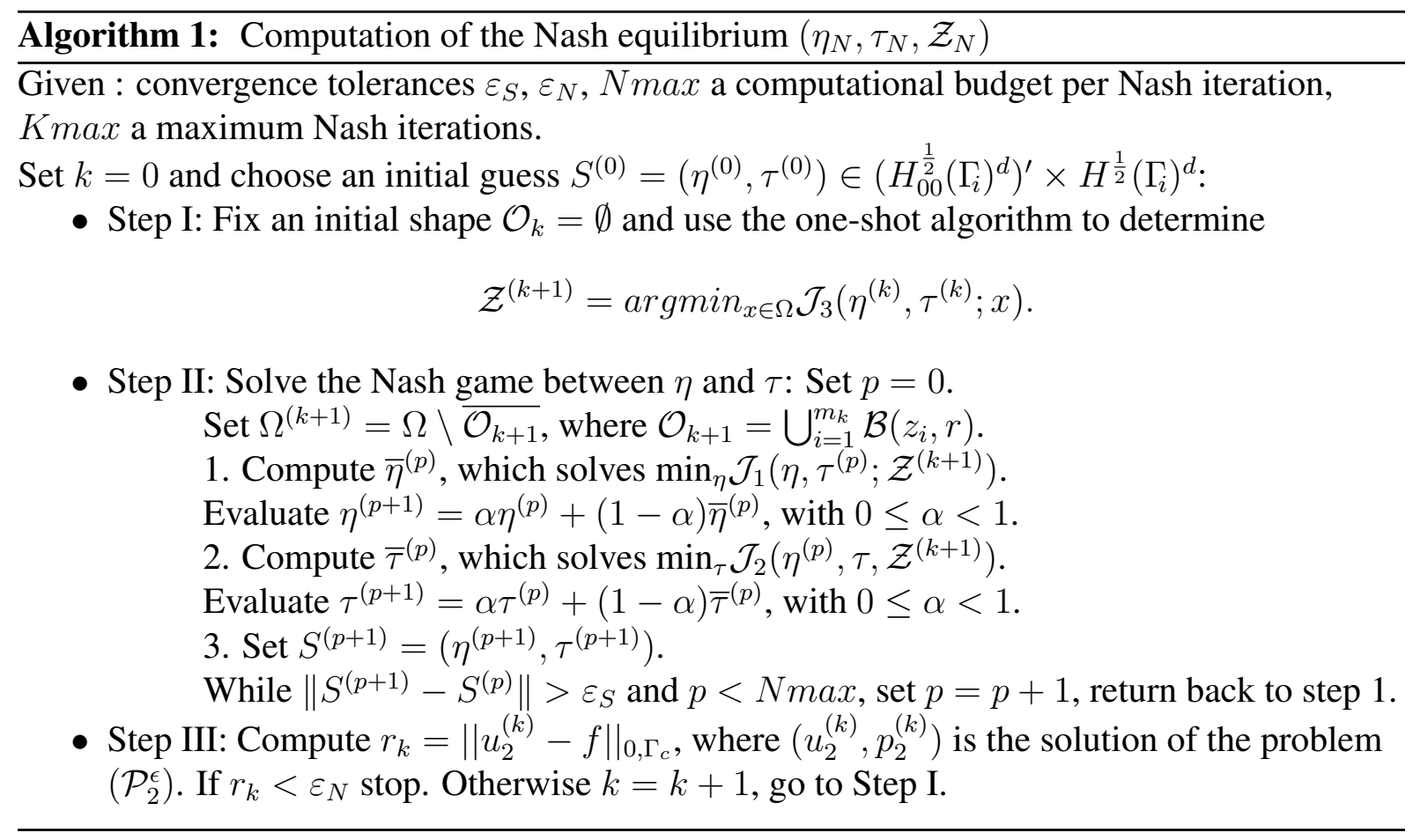

\section{NUMERICAL SIMULATIONS.}

In this section, we will present some numerical reconstructions in two dimensions to show the efficiency of our novel approach, using synthetic data generated via a finite element resolution of boundary value problem, corresponding to a homogeneous Neumann condition on the boundary $\partial \mathcal{O}_{\epsilon}^{*}$ with the code FreeFem $++[10]$.

The exterior boundary is assumed to be the rectangle $\Omega=[-0.5,0.5] \times[-0.25,0.25]$, which will be split in two components: the inaccessible part of the boundary $\Gamma_{i}=\{0.5\} \times[-0.25,0.25]$ and the accessible part $\Gamma_{c}=\partial \Omega \backslash \Gamma_{i}$, where the Cauchy data are available.

In the following, the subscripts $e x$ and opt denote, respectively, exact and optimal values.

Case A-Single object. First, we start testing the detection of a single circle $\mathcal{O}^{*}=\mathcal{C}\left(z_{\text {ex }}, r\right)$ centered at $z_{e x}$ and with radius $r$ where $z_{e x}=(-0.3,-0.15)$ and $r=0.025$.

Figure 2 presents the evolution of the three costs functionals as functions of overall Nash iterations for unnoisy data. We observe that during the early Nash iteration process, Player 1 shows a fast decrease of its cost $\mathcal{J}_{1}$ then stagnates around 0.005 , while the other players 2 and 3 continuously improve their own cost $\mathcal{J}_{2}$ and $\mathcal{J}_{3}$ respectively. The fact that each player controls only his strategy, while there is a strong dependence of each player's cost on the joint strategy, justifies the game theory framework employed to formulate the iterative negotiation between the costs. Typical of a static Nash equilibrium, the three players then proceed in the negotiation until none of them can unilaterally improve its cost, a behavior which is observed around iteration 18. The detection is quite efficient, see Figure 3(a)-(b), where 3(a) presents the iso-values of the topological gradient and 3(b) presents the obtained domain at convergence. Figure 4 show 
the reconstructed Dirichlet and Neumann boundary data. It can be seen that the reconstructed Dirichlet data give a good approximation for the exact one, while the reconstructed Neumann data deviate from the exact one, especially near the endpoints of the unspecified boundary, which is the region of singularities. At convergence, the approximate location $z_{o p t}$ is equal to $(-0.294,-0.174)$ and the relative error on Dirichlet data $\frac{\left\|\tau_{o p t}-u_{e x}\right\|_{L^{2}\left(\Gamma_{i}\right)}}{\left\|u_{e x} \mid\right\|_{L^{2}\left(\Gamma_{i}\right)}}$ and Neumann data $\frac{\left\|\eta_{o p t}-\sigma\left(u_{e x}, p_{e x}\right) n\right\|_{L^{2}\left(\Gamma_{i}\right)}}{\left\|\sigma\left(u_{e x}, p_{e x}\right) n\right\|_{L^{2}\left(\Gamma_{i}\right)}}$ are equal to 0.003 and 0.073 respectively.

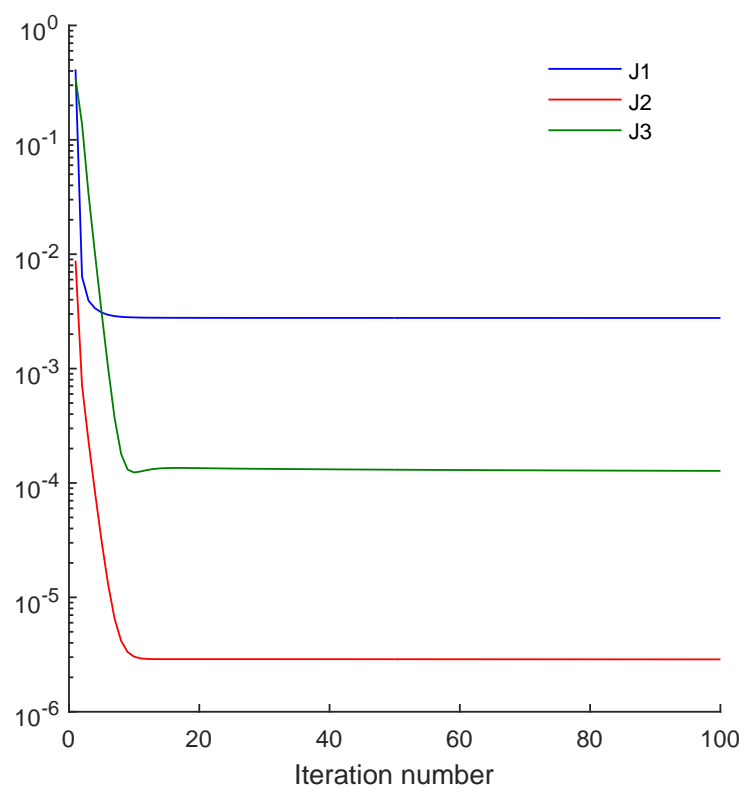

Figure 2: Case A- . Plots of the three costs $\mathcal{J}_{1}, \mathcal{J}_{2}$ and $\mathcal{J}_{3}$ as functions of overall Nash iterations

Case B-Two objects . For this test, we want to detect two circles $\mathcal{O}_{1}^{*}$ and $\mathcal{O}_{2}^{*}$ centered respectively at $(-0.4,-0.15)$ and $(-0.4,0.15)$, with shared radius $r=0.025$.

The numerical results are illustrated in Figure 5 and 6. Figure 5 shows the iso-values of the topological gradient and the obtained domain at convergence. The optimal locations are equal to $(-0.422006,-0.160662)$ and $(-0.416224,0.159772)$. In Figure 6, we present the reconstruction of the missing Dirichlet and Neumann boundary data.

\section{CONCLUSION}

In the present work, we addressed the coupled inverse problem of recovering the missing boundary data and determining the best locations of an unknown number of small objects included in a stationary viscous fluid. To treat this problem, we have proposed to formulate it as static with complete information Nash game, where the two first players target the data completion while the third one seeks to determine the number and the locations of the objects. The latter problem (identification) is formulated as a topological one. Topological sensitive analysis related to a considered Kohn-Vogelius functional has been investigated. Then we have introduced a new algorithm for computing the Nash equilibrium for three players. We have then presented two test-cases, which showed that small inclusions close to the accessible part of the boundary could be recovered, as well as the missing boundary data on the inaccessible part. 
(a)

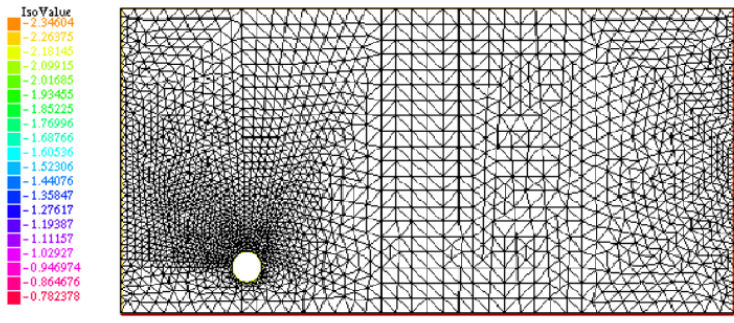

(b)

Figure 3: Case A- Detection of a single object (for unnoisy data). (a) the iso-values of the topological gradient at convergence (b) the approximate location $z_{\text {opt }}$ is equal to $(-0.294,-0.174)$, while the exact one $z_{e x}$ is equal to $(-0.3,-0.15)$.

(a)
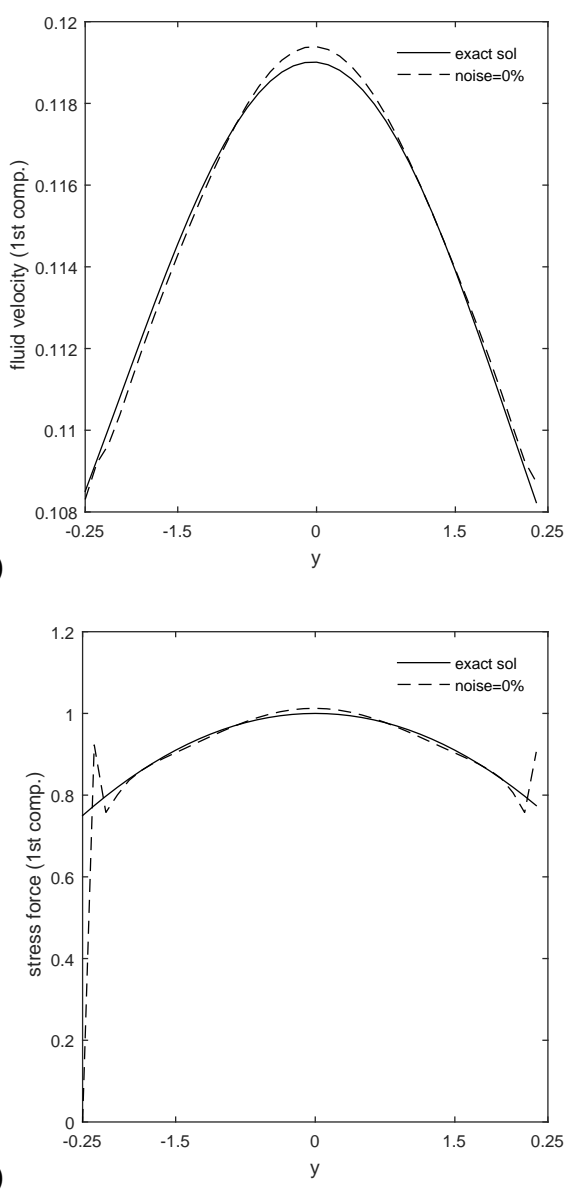

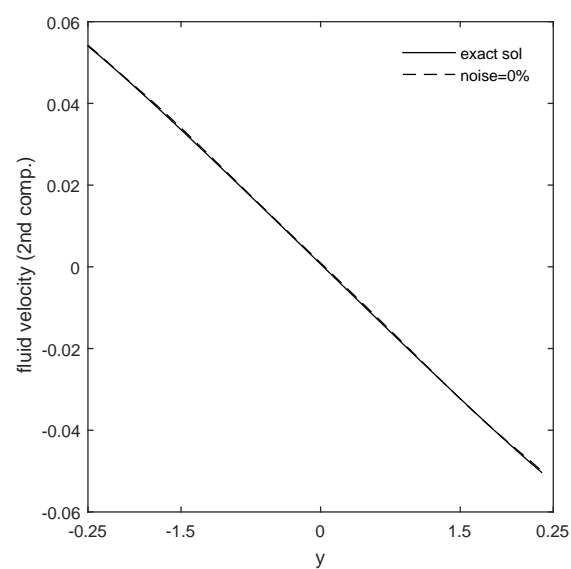

(b)

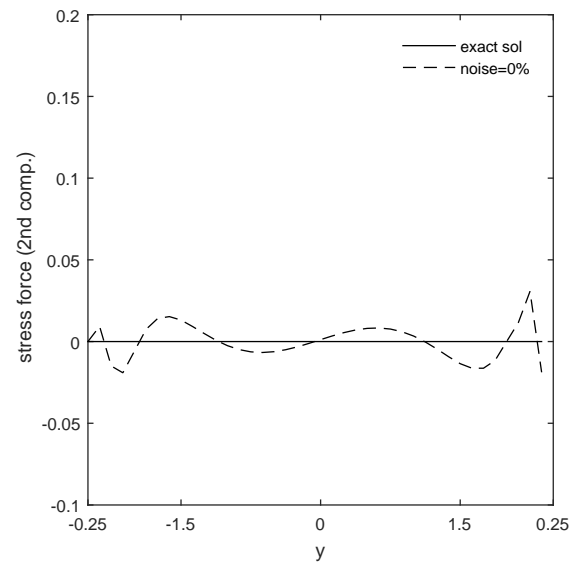

(d)

Figure 4: Case A- Reconstruction of the missing boundary data (for unnoisy data). (a) exact -line- and computed -dashed line- first component of the velocity over $\Gamma_{i}$ (b) exact -line- and computed -dashed linesecond component of the velocity over $\Gamma_{i}$ (c) exact -line- and computed -dashed line- first component of the normal stress over $\Gamma_{i}(\mathrm{~d})$ exact -line- and computed -dashed line- second component of the normal stress over $\Gamma_{i}$.

Aknowledgements. This work was financially supported by the "PHC Utique" program NAMRED of the French Ministry of Foreign Affairs and Ministry of higher education, research and innovation and the Tunisian Ministry of higher education and scientific research in the CMCU project number $18 \mathrm{G} 1502$. 

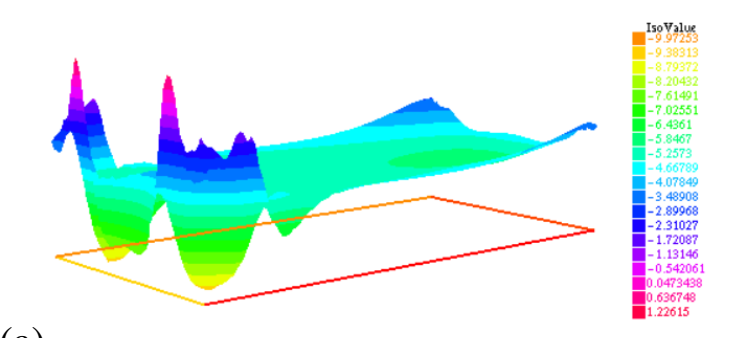

(a)

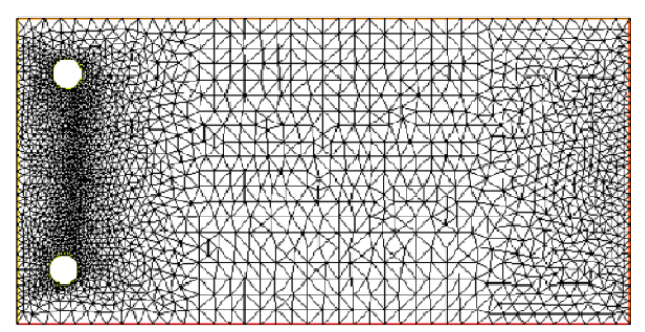

(b)

Figure 5: Case B- Detection of two objects (for unnoisy data). (a) the iso-values of the topological gradient at convergence (b) the approximate locations are equal to $z_{o p t}^{1}=(-0.422006,-0.160662)$ and $z_{\text {opt }}^{2}=(-0.416224,0.159772)$, while the exact ones are equal to $z_{\text {ex }}^{1}=(-0.4,-0.15)$ and $z_{\text {ex }}^{2}=$ $(-0.4,0.15)$.

(a)
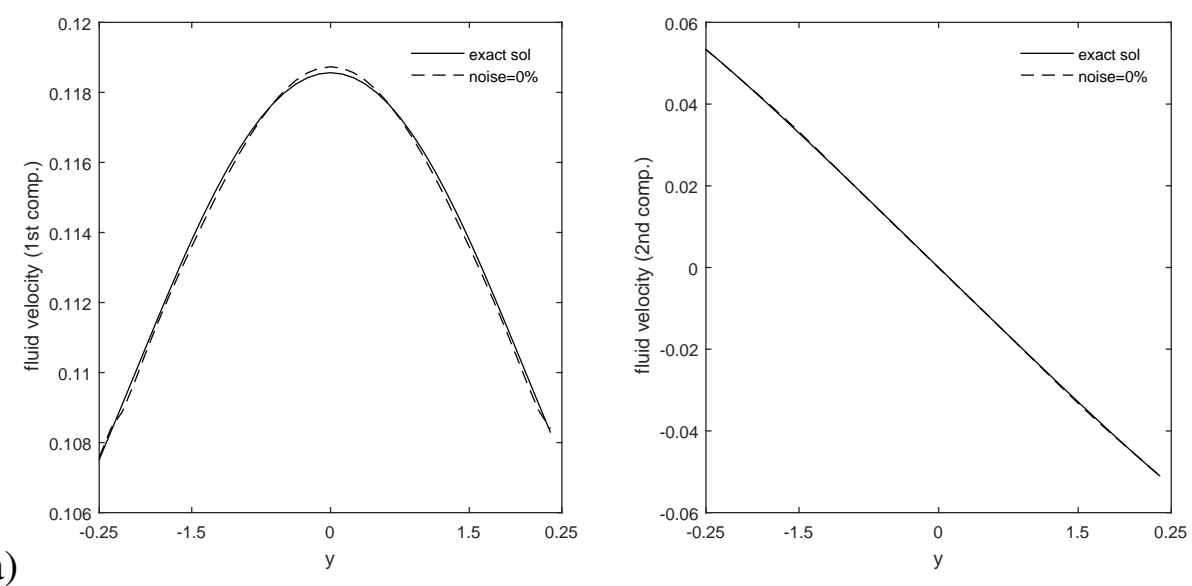

(b)
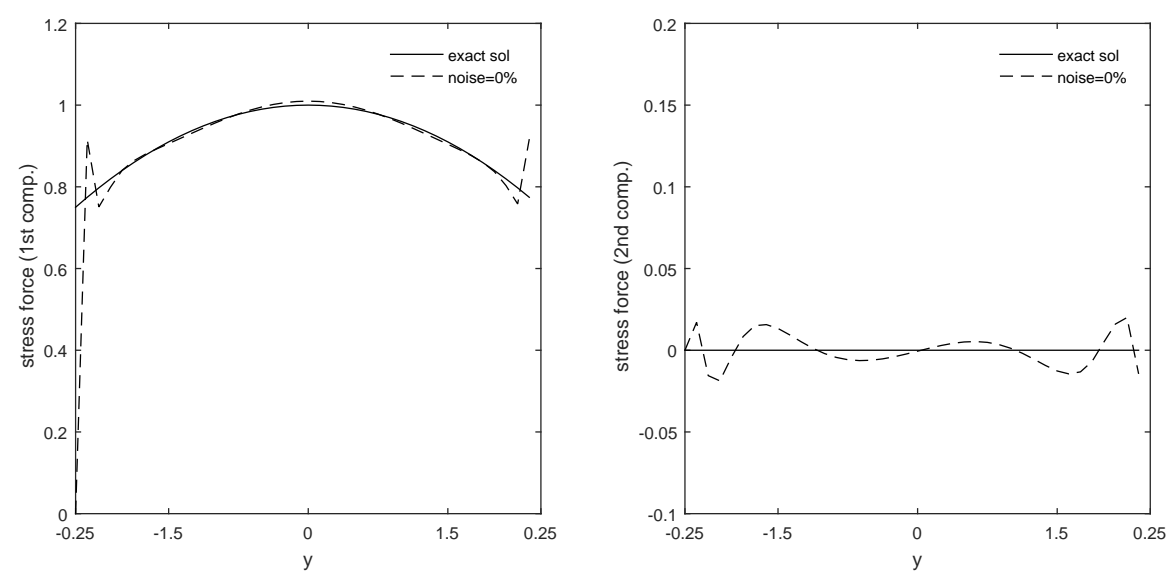

(d)

Figure 6: Case B- Reconstruction of the missing boundary data (for unnoisy data). (a) exact -line- and computed -dashed line- first component of the velocity over $\Gamma_{i}$ (b) exact -line- and computed -dashed linesecond component of the velocity over $\Gamma_{i}$ (c) exact -line- and computed -dashed line- first component of the normal stress over $\Gamma_{i}$ (d) exact -line- and computed -dashed line- second component of the normal stress over $\Gamma_{i}$.

\section{REFERENCES}

[1] G. Alessandrini, L. Rondi, E. Rosset, and S. Vessella. The stability for the Cauchy problem for elliptic equations. Inverse Problems, 25:123004, 2009. 
[2] S. Amstutz, I. Horchani, and M. Masmoudi. Crack detection by the topological gradient method. Control and Cybernetics, 34:81-101, 2005.

[3] H. Attouch, J. Bolte, P. Redont, and A. Soubeyran. Alternating proximal algorithms for weakly coupled convex minimization problems: Applications to dynamical games and PDE's. Journal of Convex Analysis, 15:485-506, 2008.

[4] A. Ben Abda, M. Hassine, M. Jaoua, and M. Masmoudi. Topological sensitivity analysis for the location of small cavities in Stokes flow. SIAM Journal on Control and Optimization, 48:2871-2900, 2009.

[5] H.A. Eschenauer, V. Kobelev, and A. Schumacher. Bubble method for topology and shape optimization of structures. Structural Optimization, 8:42-51, 1994.

[6] S. Garreau, P. Guillaume, and M. Masmoudi. The topological asymptotic for PDE systems: The elasticity case. SIAM Journal on Control and Optimization, 39:1756-1778, 2001.

[7] A. Habbal and M. Kallel. Neumann-Dirichlet Nash strategies for the solution of elliptic Cauchy problems. SIAM Journal on Control and Optimization, 51:4066-4083, 2013.

[8] A. Habbal, M. Kallel, and M. Ouni. Nash strategies for the inverse inclusion CauchyStokes problem. Inverse problems and imaging, 13:827-862, 2019.

[9] J. Hadamard. The Cauchy Problem and the Linear Hyperbolic Partial Differential Equations. Dover, New York, 1953.

[10] F. Hecht. New development in FreeFem++. Journal of Numerical Mathematics, 20:251$265,2012$.

[11] M. Masmoudi, J. Pommier, and B. Samet. The topological asymptotic expansion for the Maxwell equations and some applications. Inverse Problems, 21:547-564, 2005.

[12] J. Sokolowski and A. Zochowski. On the topological derivative in shape optimization. SIAM Journal on Control and Optimization, 37:1251-1272, 1999. 